\title{
Second Microlocalization at the Boundary and Microhyperbolicity
}

\author{
By \\ Motoo UCHIDA* and Giuseppe ZAMPIERI**
}

\begin{abstract}
The purpose of this paper is to construct the "sheaf" of 2-hyperfunctions at the boundary along an involutive submanifold and to generalize the notion of microhyperbolicity at the boundary. Let $M$ be a real analytic manifold, $X$ a complexification of $M$, and let $\Omega$ be an open subset of $M$ with $C^{\omega}$-boundary $N$. Let $V$ be a conic involutive submanifold of ${ }^{\circ}{ }_{M}^{*} X$ which intersects transversally to $N \underset{M}{\times} \stackrel{\circ}{T}_{M}^{*} X$ with regular involutive intersection. Then we define the complex of $\mathscr{E}_{X}$-Modules $\mathscr{B}_{S \mid X}^{2, V}$ of 2-hyperfunctions at the boundary along $V$, which appears to be a useful tool in studying noncharacteristic boundary value problems. Remark that the complex $\mathscr{C}_{\Omega \mid X}$ was first introduced by P. Schapira [S 3] for the microlocal study of boundary value problems. Next we introduce the notion of $\Omega$-V-hyperbolicity of a system $\mathscr{M}$ of microdifferential equations and prove that it implies "propagation of zeros up to the boundary" of cohomology groups of the complex $\mathbf{R} \mathscr{H}_{\text {o } m_{\delta_{x}}}(\mathscr{M}$, $\mathscr{B}_{\Omega \mid X}^{2}$ ). This implies in particular " $\Omega$-regularity" of $\mathscr{M}$ in the sense of [S 3$]$.
\end{abstract}

\section{$\S 1$. Microlocalization}

Let $X$ be a real $C^{2}$-manifold, $T^{*} X$ the cotangent bundle to $X, \pi: T^{*} X \rightarrow X$ the natural projection.

$D^{+}(X)$ denotes the derived category of complexes of sheaves of modules on $X$ bounded from below. Refer to $[\mathrm{H}]$ for the notion of derived categories and derived functors.

Let $M, Y$ be two closed submanifolds of $X$ with $M \subset Y$, and $A, B$ two locally closed subsets of $Y$ with $A=B \cap M$. For $\mathscr{F} \in \mathrm{Ob}\left(D^{+}(X)\right)$ we define $\mu_{A}(\mathscr{F})$, the microlocalization of $\mathscr{F}$ along $A$, by

$$
\mu_{A}(\mathscr{F})=\mu \operatorname{hom}\left(\mathbb{Z}_{A}, \mathscr{F}\right),
$$

where $\mu$ hom ( , ) is the bifunctor defined in [K-S 2] (cf.also [S 3]). We note that there are a natural morphism

Communicated by M. Kashiwara, February 23, 1988. Revised September 27, 1989.

* Department of Mathematics, Faculty of Science, University of Tokyo, Hongo, Bunkyo-ku, Tokyo, 113 Japan.

** Seminario Matematico, Università di Padova, Via Belzoni, 7, 35131 Padova, Italy. 


$$
\mu_{A}(\mathscr{F}) \longrightarrow \mathbf{R} \Gamma_{M_{X} T^{*} X}\left(\mu_{B}(\mathscr{F})\right)
$$

and an isomorphism

$$
\begin{aligned}
\mathbf{R} \pi_{*} \mu_{A}(\mathscr{F}) & \simeq \mathbf{R} \pi_{*} \mathbf{R} \Gamma_{M_{X} T^{*} X}\left(\mu_{\beta}(\mathscr{F})\right) \\
& \cong \mathbf{R} \Gamma_{A}(\mathscr{F}) .
\end{aligned}
$$

Thus we have a commutative diagram

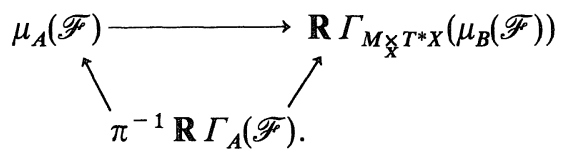

Refer to [K-S 2] for the details about microlocalization, functors and the notation that we use in this paper.

\section{§2. The Complex $\mathscr{C}_{\Omega \mid X}^{2}$}

In this section we assume that $M$ is a real analytic manifold of product type $M=M^{\prime} \times L$ with complexification $X=X^{\prime} \times Z$ and dimension $n=n_{1}$ $+n_{2}$. We denote by $\mathcal{O}_{X}$ the sheaf of holomorphic functions on $X$, and $\mathscr{E}_{X}$ the sheaf of microdifferential operators on $T^{*} X$. For a locally closed set $A^{\prime} \subset M^{\prime}$, we put $A=A^{\prime} \times L$ and define

$$
\begin{gathered}
\mathscr{C}_{A \mid X}^{h}=\mu_{A^{\prime} \times Z}\left(\mathcal{O}_{X}\right) \otimes \omega_{M^{\prime} / X^{\prime}}\left[n_{1}\right], \\
\mathscr{C}_{A \mid X}^{2}=\mu_{T^{*} X^{\prime} \times L}\left(\mathscr{C}_{A \mid X}^{h}\right) \otimes \omega_{L / Z}\left[n_{2}\right], \\
\mathscr{B}_{A \mid X}^{2}=\left.\mathscr{C}_{A \mid X}^{2}\right|_{T^{*} X^{\prime} \times L}=\mathbf{R} \Gamma_{T^{*} X^{\prime} \times L}\left(\mathscr{C}_{A \mid X}^{h}\right) \otimes \omega_{L / Z}\left[n_{2}\right],
\end{gathered}
$$

with $\omega_{M^{\prime} / X^{\prime}}, \omega_{L / Z}$ being the relative orientation sheaves (cf. [S 3], [S 4]). $\mathscr{C}_{A \mid X}^{h}$ and $\mathscr{B}_{A \mid X}^{2}$ are complexes of $\pi^{-1} \mathscr{D}_{X}$-modules on $T^{*} X^{\prime} \times Z$ and $T^{*} X^{\prime} \times L$ respectively, and $\mathscr{C}_{A \mid X}^{2}$ is a complex of $\pi_{L}^{-1} \pi^{-1} \mathscr{D}_{X}$-modules on $T^{*} X^{\prime} \times T_{L}^{*} Z$ $\left(\pi_{L}: T^{*} X^{\prime} \times T_{L}^{*} Z \rightarrow T^{*} X^{\prime} \times L\right) . \quad X$ being the complexification of $M$, we identify $M \underset{X}{\times} T^{*} X$ and $T_{M}^{*} X \underset{M}{\bigoplus} T^{*} M$ in the following statements of this section. Let $p \in T_{M^{\prime}}^{*} X^{\prime} \times L, \pi(p)=x$.

Theorem 2.1. Let $A=A^{\prime} \times L$ be an open subset (resp. a closed subset) of $M=M^{\prime} \times L$ such that

$$
N_{x}^{*}(A) \neq T_{x}^{*} M .
$$

Then any germ $\phi$ of complex contact transformation at $p$ preserving $T_{M^{\prime}}^{*} X^{\prime} \times L$ 
and $\left(\bar{A} \underset{M}{\times} T_{M}^{*} X\right) \underset{M}{\bigoplus} N^{*}(A)^{a}$ (resp. $\left.\left(A \underset{M}{\times} T_{M}^{*} X\right) \underset{M}{\bigoplus} N^{*}(A)\right)$ may be quantized to quasi-isomorphisms of complexes

$$
\mathscr{C}_{A \mid X, p}^{h} \cong \mathscr{C}_{A \mid X, \phi(p)}^{h}, \quad \mathscr{B}_{A \mid X, p}^{2} \cong \mathscr{B}_{A \mid X, \phi(p)}^{2} .
$$

Proof. We first note that $\phi$ preserves $\operatorname{Int}(A) \underset{M}{\times} T_{M}^{*} X$, the $I$-symplectic regular part of $\left(\bar{A} \underset{M}{\times} T_{M}^{*} X\right) \underset{M}{\oplus} N^{*}(A)^{a}$. Thus $\phi$ preserves

$$
\begin{aligned}
\Lambda_{1}= & {\left[\left(\bar{A} \underset{M}{\times} T_{M}^{*} X\right) \underset{M}{\oplus} N^{*}(A)^{a}\right] \cap\left(T^{*} X^{\prime} \times Z\right) } \\
= & {\left[\left(\bar{A}^{\prime} \underset{M^{\prime}}{\times} T_{M^{\prime}}^{*} X^{\prime}\right) \underset{M^{\prime}}{\oplus} N^{*}\left(A^{\prime}\right)^{a}\right] \times L, } \\
\Lambda_{2}= & {\left[\left(\bar{A}^{\prime} \times{ }_{M^{\prime}}^{\times} T_{M^{\prime}}^{*} X^{\prime}\right) \underset{M^{\prime}}{\oplus} N^{*}\left(A^{\prime}\right)^{a}\right] \times Z } \\
= & \text { the union of complex bicharacteristic leaves of } T^{*} X^{\prime} \times Z \text { issued } \\
& \text { from } \Lambda_{1}, \\
\Lambda_{10}= & \left(\operatorname{Int}\left(A^{\prime}\right) \underset{M^{\prime}}{\times} T_{M^{\prime}}^{*} X^{\prime}\right) \times L \\
= & \left(\operatorname{Int}(A) \underset{M}{\times} T_{M}^{*} X\right) \cap \Lambda_{1},
\end{aligned}
$$

and

$$
\begin{aligned}
\Lambda_{20}= & \left(\operatorname{Int}\left(A^{\prime}\right) \underset{M^{\prime}}{\times} T_{M^{\prime}}^{*} X^{\prime}\right) \times Z \\
= & \text { the union of complex bicharacteristic leaves of } T^{*} X^{\prime} \times Z \text { issued } \\
& \quad \text { from } \Lambda_{10} .
\end{aligned}
$$

Now let $\Phi: D^{+}(X ; p) \rightarrow D^{+}(X ; \phi(p))$ be a quantized contact transformation over $\phi$ with shift $n$ (cf. [K-S 2]). Since for $A$ open (resp. closed) $\operatorname{SS}\left(Z_{A^{\prime} \times Z}\right)$ ( = the microsupport of the sheaf $\mathbf{Z}_{A^{\prime} \times Z}$ on $X$ ) $\subset \Lambda_{2}$ (resp. $\subset \Lambda_{2}^{a}$ ) and the sheaf $\mathbb{Z}_{A^{\prime} \times X}$ is simple with shift $\frac{1}{2} n_{1}$ on $\Lambda_{20}$, by Lemma 2.2 below (Cor. 1.2 of [U 2]),

$$
\Phi\left(\mathbf{Z}_{A^{\prime} \times Z}\right) \cong \mathbf{Z}_{A^{\prime} \times Z} \text { in } D^{+}(X ; \phi(p)) .
$$

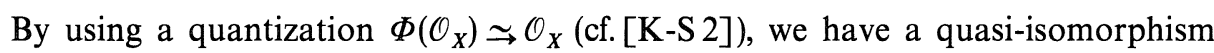

$$
\mathscr{C}_{A \mid X, p}^{h} \cong \mathscr{C}_{A \mid X, \phi(p)}^{h}
$$

This induces also a quasi-isomorphism on $\mathscr{B}_{A \mid X}^{2} \simeq \mathbf{R} \Gamma_{\Lambda_{1}}\left(\mathscr{C}_{A \mid X}^{h}\right)\left[n_{2}\right]$.

Lemma 2.2. (cf. [U 2]). Let $X$ be a $C^{2}$-manifold, $Y$ a closed submanifold of 
$X$, and $B$ an open (resp. closed) subset of $Y$ such that $N_{x}^{*}(B) \neq T_{x}^{*} Y$. Suppose that $\mathscr{F} \in \mathrm{Ob}\left(D^{+}(X)\right)$ be simple with shift $\frac{1}{2} \operatorname{codim} Y$ on $\operatorname{Int}(B) \underset{Y}{\times} T_{Y}^{*} X$ and, in a neighborhood of $p \in\left(T_{Y}^{*} X\right)_{x}$,

$$
\mathrm{SS}(\mathscr{F}) \subset \varpi_{Y} \rho_{Y}^{-1}\left(\bar{B} \underset{Y}{\times} N^{*}(B)^{a}\right)\left(\text { resp. SS }(\mathscr{F}) \subset \varpi_{Y} \rho_{Y}^{-1}\left(B \underset{Y}{\times} N^{*}(B)\right)\right.
$$

with $\varpi_{Y}, \rho_{Y}$ being the natural mappings $T^{*} Y \underset{\rho_{Y}}{\stackrel{Y}{\underset{X}{x}}} T^{*} X \underset{\varpi_{Y}}{\longrightarrow} T^{*} X$ associated to $Y \hookrightarrow X$. Then $\mathscr{F}$ is microlocally isomorphic to $\mathbb{Z}_{B}$ at $p$.

Proof. By Prop. 6.2.1 of [K-S 2] it is not restrictive to assume

$$
\mathscr{F} \simeq \mathscr{F}_{Y} .
$$

We have $\operatorname{SS}\left(\left.\mathscr{F}\right|_{Y}\right) \subset N^{*}(B)^{a}\left(\right.$ resp. $\left.\operatorname{SS}\left(\left.\mathscr{F}\right|_{Y}\right) \subset N^{*}(B)\right)$ at $p$, and therefore

$$
\left.\mathscr{F}\right|_{Y} \simeq\left(\left.\mathscr{F}\right|_{Y}\right)_{B} \quad\left(\text { resp. }\left.\mathscr{F}\right|_{Y} \simeq \mathbb{R} \Gamma_{B}^{\circ}\left(\left.\mathscr{F}\right|_{Y}\right)\right)
$$

We observe now that, for a system of neighborhoods $U$ of $x, U \cap B$ is contractible due to (2.4). From this and from the simpleness of $\mathscr{F}$ in $\stackrel{\circ}{B} \underset{Y}{\times} T_{Y}^{*} X$, we get

$$
\mathbb{R} \Gamma_{B}^{\circ}\left(\left.\mathscr{F}\right|_{Y}\right) \simeq \mathbb{Z}_{\bar{B}}
$$

From (2.6)-(2.8) the conclusion follows.

We choose now $A=M$ in (2.1)-(2.3). Then $\mathscr{C}_{M \mid X}^{2}$ (resp. $\mathscr{B}_{M \mid X}^{2}$ ) is nothing but the sheaf of Kashiwara's 2-microfunctions (resp. 2-hyperfunctions) along $V$ $=\stackrel{\circ}{T}_{M^{\prime}}^{*} X^{\prime} \times L($ cf. $[K],[\mathrm{K}-\mathrm{L}])$. The complex $\mathscr{C}_{M \mid X}^{2}\left(\right.$ resp. $\left.\mathscr{B}_{M \mid X}^{2}\right)$ is concentrated in degree 0 and intrinsically defined on $T_{V}^{*} \widetilde{V} \simeq \stackrel{\circ}{T}_{M^{\prime}}^{*} X^{\prime} \times T_{L}^{*} Z$ (resp. $V$ ); moreover the canonical morphism

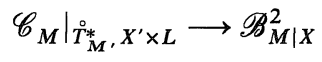

is injective, where $\mathscr{C}_{M}$ is the sheaf of Sato's microfunctions.

Next we consider the complexes $\mathscr{B}_{N \mid X}^{2}$ and $\mathscr{C}_{N \mid X}^{2}$ for a closed analytic submanifold $N=N^{\prime} \times L$ of $M=M^{\prime} \times L$ of codimension $d \geq 1 . \quad \mathscr{B}_{N \mid X}^{2}\left(\right.$ resp. $\left.\mathscr{C}_{N \mid X}^{2}\right)$ is concentrated in degree 0 and intrinsically defined on $\stackrel{\circ}{N}_{N^{\prime}}^{*} X^{\prime} \times L$ (resp. on $\left.T_{T_{N}^{*}, X^{\prime} \times L}^{*}\left(\stackrel{\circ}{T}_{N^{\prime}}^{*} X^{\prime} \times Z\right) \cong \stackrel{\circ}{T}_{N^{\prime}}^{*} X^{\prime} \times T_{L}^{*} Z\right)$. Moreover there is a natural injective morphism

$$
\left.\mathscr{C}_{N \mid X}\right|_{T_{N}^{*}, X^{\prime} \times L} \longrightarrow \mathscr{B}_{N \mid X}^{2}\left(\mathrm{cf} \text {. [K-K] as for } \mathscr{C}_{N \mid X}\right)
$$

The injectivity of this morphism can be proved by reducing it to that of the morphism $\left.\mathscr{C}_{M}\right|_{V} \rightarrow \mathscr{B}_{V}^{2}((2.9))$.

We now describe the stalks of $\mathscr{C}_{N \mid X}^{2}, \mathscr{C}_{M \mid X}^{2}$ by means of cohomology groups 
of $\mathcal{O}_{X}$ in degree 1 . We take a system of local coordinates

$$
\begin{aligned}
& z=\left(z^{\prime}, z^{\prime \prime}, z_{n_{1}}\right) \in \mathbb{C}^{d} \times \mathbb{C}^{n_{1}-d-1} \times \mathbb{C}^{1} \simeq X^{\prime} \text { with } Y^{\prime}=N^{\prime C}=\left\{z^{\prime}=0\right\}, \\
& w=\left(w^{\prime}, w_{n_{2}}\right) \in \mathbb{C}^{n_{2}-1} \times \mathbb{C}^{1} \simeq Z, \\
& (z, w ; \zeta, \tau) \in T^{*} X \simeq T^{*} X^{\prime} \times T^{*} Z .
\end{aligned}
$$

We set

$$
\begin{aligned}
& G_{M}^{\prime}=\left\{z \in X^{\prime} ; \operatorname{Im} z_{n_{1}} \leqq\left(\operatorname{Im} z^{\prime}\right)^{2}+\left(\operatorname{Im} z^{\prime \prime}\right)^{2}\right\}, \quad G_{M}=G_{M}^{\prime} \times Z, \\
& G_{N}^{\prime}=\left\{z \in X^{\prime} ; \operatorname{Im} z_{n_{1}} \leqq\left(\operatorname{Im} z^{\prime \prime}\right)^{2}\right\}, \quad G_{N}=G_{N}^{\prime} \times Z, \\
& D=\left\{w \in Z ; \operatorname{Im} w_{n_{2}}>\left(\operatorname{Im} w^{\prime}\right)^{2}\right\},
\end{aligned}
$$

and take a point

$$
p=\left(p_{1}, p_{2}\right) \in\left(N_{M^{\prime}}^{\times} T_{M^{\prime}}^{*} X^{\prime}\right) \times T_{L}^{*} Z \text { with } \zeta_{n_{1}} \neq 0, \tau_{n_{2}} \neq 0 \text { at } p
$$

With these notations we introduce contact transformations $\phi_{1}$ on $T^{*} X^{\prime} \backslash T_{Y^{\prime}}^{*} X^{\prime}$ and $\phi_{2}$ on $\stackrel{\circ}{T}^{*} Z$ which transform

$$
\phi_{1}\left(T_{M^{\prime}}^{*} X^{\prime}\right)=T_{\partial G_{M}^{\prime}}^{*} X^{\prime}, \quad \phi_{1}\left(T_{N^{\prime}}^{*} X^{\prime}\right)=T_{\partial G_{N}^{\prime}}^{*} X^{\prime}, \phi_{2}\left(T_{L}^{*} Z\right)=T_{\partial D}^{*} Z,
$$

and

$$
\phi_{1}\left(p_{1}\right)=\left(0 ; i d z_{n_{1}}\right), \quad \phi_{2}\left(p_{2}\right)=\left(0 ; i d w_{n_{2}}\right) .
$$

We then quantize $\phi_{1}$ on $T^{*} X$ and thus get isomorphisms

$$
\begin{aligned}
& \left.\phi_{1 *}\left(\mathscr{C}_{M \mid X}^{h}\right) \simeq \mathscr{H}_{G_{M}}^{1}\left(\mathcal{O}_{X}\right)\right|_{\partial G_{M}}, \\
& \left.\phi_{1 *}\left(\mathscr{C}_{N \mid X}^{h}\right) \simeq \mathscr{H}_{G_{N}}^{1}\left(\mathcal{O}_{X}\right)\right|_{\partial G_{N}},
\end{aligned}
$$

where we identify $\left(\stackrel{\circ}{T}_{M^{\prime}}^{*} X^{\prime} \times Z, \stackrel{\circ}{T}_{N^{\prime}}^{*} X^{\prime} \times Z\right)$ and $\left(\stackrel{\circ}{T}_{\partial G_{M}}^{*} X, \stackrel{\circ}{T}_{\partial G_{N}}^{*} X\right)$ via $\phi_{1} \times \mathrm{id}_{T^{*} Z}$ from a neighborhood of $\left(p_{1},(\mathrm{w} ; 0)\right)$ to a neighborhood of $\left(\phi_{1}\left(p_{1}\right),(w ; 0)\right)$. Next we quantize $\phi_{2}$ on $T^{*}\left(\stackrel{\circ}{T}_{\partial}^{*} G_{M}^{\prime} X^{\prime} \times Z\right) \simeq T^{*} \stackrel{\circ}{T}_{\partial G_{M}^{\prime}}^{*} X^{\prime} \times T^{*} Z$ and get

$$
\begin{aligned}
\mathscr{C}_{M \mid X, p}^{2} \cong & \mathscr{H}_{\partial G_{M}^{\prime} \times(Z \backslash D)}^{1}\left(\left.\mathscr{H}_{G_{M}}^{1}\left(\mathcal{O}_{X}\right)\right|_{\partial G_{M}^{\prime} \times Z}\right)_{(0,0)} \\
\cong & \frac{\frac{\lim _{W}}{W} \Gamma\left(W \cap\left(\partial G_{M}^{\prime} \times D\right),\left.\mathscr{H}_{G_{M}}^{1}\left(\mathcal{O}_{X}\right)\right|_{\partial G_{M}^{\prime} \times Z}\right)}{\underline{\lim _{W}} \Gamma\left(W \cap\left(\partial G_{M}^{\prime} \times Z\right),\left.\mathscr{H}_{G_{M}}^{1}\left(\mathcal{O}_{X}\right)\right|_{\partial G_{M}^{\prime} \times Z}\right)} \\
\cong & \frac{\lim _{W, W_{M}}}{\longrightarrow} H_{G_{M}}^{1}\left(W_{M}, \mathcal{O}_{X}\right) / \frac{\varliminf_{W}}{W} H_{G_{M}}^{1}\left(W, \mathcal{O}_{X}\right)
\end{aligned}
$$

for $W\left(\right.$ resp. $\left.W_{M}\right)$ ranging through the family of neighborhoods of $(0,0)$ (resp. of 
$W \cap\left(\partial G_{M}^{\prime} \times D\right)$ ). (In doing the above calculation one has only to remark that $\left.\Gamma_{G_{M}}\left(\mathcal{O}_{X}\right)\right|_{\partial G_{M}}=0$.) We refer to [S-Z 2] and [U 1] for the above quantization with respect to holomorphic parameters. At the next step we replace by excision $W_{M}$ with $W_{M}^{\prime}=W_{M} \cup\left(\left(X \backslash G_{M}\right) \cap W\right)$ in (2.15). We also notice that the sequence

$$
H_{G_{M}}^{1}\left(W, \mathcal{O}_{X}\right) \longrightarrow H_{G_{M}}^{1}\left(W_{M}^{\prime}, \mathcal{O}_{X}\right) \longrightarrow H^{1}\left(W_{M}^{\prime}, \mathcal{O}_{X}\right) \longrightarrow 0,
$$

is exact. Thus from (2.15) we obtain our basic representation

$$
\mathscr{C}_{M \mid X, p}^{2} \cong \varliminf_{W_{M}^{\prime}} H^{1}\left(W_{M}^{\prime}, \mathcal{O}_{X}\right)
$$

In similar way one proves that

$$
\begin{aligned}
\mathscr{C}_{N \mid X, p}^{2} & \cong \mathscr{H}_{\partial G_{N \times(Z \backslash D)}^{1}}^{1}\left(\left.\mathscr{H}_{G_{N}}^{1}\left(\mathcal{O}_{X}\right)\right|_{\partial G_{N}^{\prime} \times Z}\right)_{(0,0)} \\
& \cong \varliminf_{W_{N}^{\prime}} H^{1}\left(W_{N}^{\prime}, \mathcal{O}_{X}\right)
\end{aligned}
$$

for $W_{N}^{\prime}$ varying in the family of open neighborhoods of $\left(\left(\partial G_{N}^{\prime} \times D\right) \cup\right.$ $\left.\left(X \backslash G_{N}\right)\right) \cap W$. We note that the restriction from $W_{N}^{\prime}$ to $W_{M}^{\prime}$ induces a morphism

$$
\varliminf_{W_{N}^{\prime}} H^{1}\left(W_{N}^{\prime}, \mathcal{O}_{X}\right) \longrightarrow \varliminf_{W_{M}^{\prime}} H^{1}\left(W_{M}^{\prime}, \mathcal{O}_{X}\right) .
$$

Lemma 2.3. The morphism (2.18) is injective

Proof. Let $f$ be a $\bar{\partial}$-closed $(0,1)$-form with coefficients in $\Gamma\left(W_{N}^{\prime}, \mathscr{B}_{X^{R}}\right), \mathscr{B}_{X^{R}}$ being the sheaf of Sato's hyperfunctions on $X^{R} \simeq \mathbb{R}^{2 n}$. Assume that there exists a solution $u$ of the system

$$
\bar{\partial} u=f, \quad u \in \Gamma\left(W_{M}^{\prime}, \mathscr{B}_{X^{R}}\right) .
$$

Since $X \backslash G_{N}$ is Stein, we can solve in a neighborhood of 0

$$
\bar{\partial} w=f, \quad w \in \Gamma\left(X \backslash G_{N}, \mathscr{B}_{X^{R}}\right) .
$$

Thus $u-w$ is holomorphic in $X \backslash G_{M}$ and also in $\left\{(z, w) \in X \times Z\right.$; $\operatorname{Im} z_{n_{1}}$ $\left.>\left(\operatorname{Im} z^{\prime \prime}\right)^{2}, \operatorname{Im} w_{n_{2}}-\left(\operatorname{Im} w^{\prime}\right)^{2}=\varepsilon,\left|\operatorname{Im} z^{\prime}\right|<\delta\right\}\left(\forall \varepsilon \ll 1\right.$ and for $\left.\delta=\delta_{\varepsilon}\right)$. Applying the Bochner's theorem to $u-w$ in the variables $\left(z^{\prime}, w_{n_{2}}\right)$ one then sees that $u$ extends uniquely to $X \backslash G_{N}$ as a solution of $\bar{\partial} u=f$.

Next by the same argument in the variables $\left(z^{\prime}, z_{n_{1}}\right)$ one proves that $u$ extends also to a neighborhood of $\partial G_{N} \cap\left(X^{\prime} \times D\right)$ as a solution of $\bar{\partial} u=f$. In conclusion $f$ is exact in a set of type $W_{N}^{\prime}$ which proves the lemma.

We note that by applying $\mu_{T^{*} X^{\prime} \times L}() \otimes \omega_{L / Z}\left[n_{2}\right]\left(\operatorname{resp} \cdot \mathbb{R} \Gamma_{T^{*} X^{\prime} \times L}() \otimes\right.$ $\left.\omega_{L / Z}\left[n_{2}\right]\right)$ to the natural morphism

$$
\mathscr{C}_{N \mid X}^{h} \longrightarrow \mathscr{C}_{M \mid X}^{h}
$$


we get a morphism

$$
\mathscr{C}_{N \mid X}^{2} \longrightarrow \mathscr{C}_{M \mid X}^{2}
$$

(resp.

$$
\mathscr{B}_{N \mid X}^{2} \longrightarrow \mathscr{B}_{M \mid X}^{2}
$$

This morphism is clearly compatible with (2.18).

Theorem 2.4. Let $N=N^{\prime} \times L$ be a closed $C^{\omega}$-submanifold of $M=M^{\prime} \times L$.

(i) The morphism (2.20) is injective on $\left(N^{\prime} \underset{M^{\prime}}{\times} T_{M^{\prime}}^{*} X^{\prime}\right) \times T_{L}^{*} Z$. In particular $\left.\left.\mathscr{B}_{N \mid X}^{2}\right|_{\left(N^{\prime} \times M^{\prime} T_{M}^{*}, X^{\prime}\right) \times L} \rightarrow \mathscr{B}_{M \mid X}^{2}\right|_{\left(N^{\prime} \times T^{\prime} T_{M}^{*}, X^{\prime}\right) \times L}$ is injective.

(ii) Sections of $\mathscr{B}_{N \mid X}^{2}, \mathscr{C}_{N \mid X}^{2}$ have the unique continuation property along the complex bicharacteristic leaves of $Y^{\prime} \underset{X^{\prime}}{\times} T^{*} X^{\prime}$.

Proof. Consider the commutative diagram with exact rows:

$$
\begin{gathered}
0 \longrightarrow\left(\mathscr{C}_{N \mid X}^{h}\right)_{T_{N}^{*}, X^{\prime} \times L} \\
\downarrow \\
\downarrow \\
0 \longrightarrow\left(\mathscr{C}_{M \mid X}^{h}\right)_{T_{M}^{*}, X^{\prime} \times L}^{2} \longrightarrow \stackrel{\circ}{\pi}_{L *} \mathscr{C}_{N \mid X}^{2} \longrightarrow 0 \\
\downarrow \mathscr{B}_{M \mid X}^{2} \longrightarrow \stackrel{\circ}{\pi}_{L *} \mathscr{C}_{M \mid X}^{2} \longrightarrow 0
\end{gathered}
$$

$\left(\stackrel{\circ}{\pi}_{L}\right.$ being the projection $\left.T^{*} X^{\prime} \times \stackrel{\circ}{T}_{L}^{*} Z \rightarrow T^{*} X^{\prime} \times L\right)$. Thus it is enough to prove the theorem for $\mathscr{C}_{N \mid X}^{2}$ on $T^{*} X^{\prime} \times \stackrel{\circ}{T}_{L}^{*} Z$. We use now the trick of the dummy variable due to Kashiwara. We put $\hat{M}^{\prime}=M^{\prime} \times \mathbf{R}, \hat{X}^{\prime}=X^{\prime} \times \mathbf{C}, \hat{Y}^{\prime}$ $=Y^{\prime} \times \mathbf{C}$, and set $\hat{M}=\hat{M}^{\prime} \times L$ and so on. We denote

$$
T^{*} X \stackrel{\rho}{\longleftarrow} T^{*} X \times \sqrt{-1} T^{*} \mathbf{R} \stackrel{\varpi}{\longrightarrow} T^{*} \hat{X},
$$

and denote by $t$ the new variable in $\mathbf{R}$ (or $\mathbf{C}$ ). Then we have an exact commutative diagram in $T^{*} X$

$$
\begin{gathered}
0 \longrightarrow \hat{\varpi}_{*} \hat{\rho}^{-1} \mathscr{C}_{N \mid X}^{2} \stackrel{\otimes \delta_{t}}{\longrightarrow} \mathscr{C}_{\hat{N} \mid \hat{X}}^{2} \stackrel{t}{\longrightarrow} \mathscr{C}_{\hat{N} \mid \hat{X}}^{2} \longrightarrow 0 \\
\downarrow \\
0 \longrightarrow \hat{\varpi}_{*} \hat{\rho}^{-1} \mathscr{C}_{M \mid X}^{2} \stackrel{\otimes \delta_{t}}{\longrightarrow} \mathscr{C}_{\hat{M} \mid \hat{X}}^{2} \stackrel{t}{\longrightarrow} \mathscr{C}_{\hat{M} \mid \hat{X}}^{2} \longrightarrow 0
\end{gathered}
$$

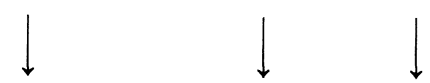

The rows of this diagram are obtained by microlocalizing the short exact sequence

$$
0 \longrightarrow \mathcal{O}_{\hat{X}} \stackrel{t}{\longrightarrow} \mathcal{O}_{\hat{X}} \longrightarrow j_{*} \mathcal{O}_{X} \longrightarrow 0,
$$


where $j$ denotes the embedding $X \hookrightarrow \hat{X}$. Because of (2.22) and (2.23) it is enough to prove the theorem in $\left(T^{*} X^{\prime} \backslash T_{Y^{\prime}}^{*} X^{\prime}\right) \times i_{L}^{*} Z$. Then (i) follows from Lemma 2.3. We prove now (ii). As seen in (2.17) we can identify

$$
\mathscr{C}_{N \mid X}^{2} \simeq \mathscr{H}_{\partial G_{N}^{\prime} \times(Z \backslash D)}^{1}\left(\left.\mathscr{H}_{G_{N}}^{1}\left(\mathcal{O}_{X}\right)\right|_{\partial G_{N}}\right)
$$

via quantization of contact transformations $\phi_{1}$ on $\left(T^{*} X^{\prime} \backslash T_{Y^{\prime}}^{*} X^{\prime}\right) \times \stackrel{\circ}{T}^{*} Z$ and $\phi_{2}$ on $T^{*}\left(\stackrel{\circ}{T}_{\partial G_{N}^{\prime}}^{*} X^{\prime}\right) \times \stackrel{\circ}{T} * Z$. (Here we are identifying $\stackrel{\circ}{T}_{T^{*}, X^{\prime} \times L}^{*}\left(T_{N^{\prime}}^{*} X^{\prime} \times Z\right.$ ) and $\stackrel{\circ}{T}_{T^{*} \partial G_{N}^{\prime} X^{\prime} \times \partial D}^{*}\left(T_{\partial G_{N}^{\prime}}^{*} X^{\prime} \times Z\right)$ via $\phi_{2}\left(\left.\phi_{1}\right|_{T_{N}^{*} X^{\prime} \times T_{T}^{*} Z}\right)$ in a neighborhood of $p$.) We set $G=\left\{\left(z^{\prime \prime}, z_{n_{1}}\right) \in \mathbb{C}^{n_{1}-d} ; \operatorname{Im} z_{n_{1}} \leqq\left(\operatorname{Im} z^{\prime \prime}\right)^{2}\right\}$. For a complex manifold $W$, we define a sheaf $\mathscr{F}_{W}$ of $\mathcal{O}_{W}$-modules on $\partial G \times W$ by

$$
\mathscr{F}_{W}=\left.\mathscr{H}_{G \times W}^{1}\left(\mathcal{O}_{\mathrm{C}^{n_{1}-d \times W}}\right)\right|_{\partial G \times W} .
$$

Then (2.24) can be rewritten as

$$
\mathscr{C}_{N \mid X}^{2} \simeq \mathscr{H}_{\partial{ }^{G \times C^{d} \times(Z \mid D)}}^{1}\left(\mathscr{F}_{\left.C^{d} \times Z\right)}\right) .
$$

In order to prove (ii) we use the following lemma, a conclusion of the abstract edge of the wedge theorem due to Kashiwara-Laurent ([K-L]).

Lemma 2.5. Suppose that we are given a contravariant functor which associates to each complex manifold $W$ a sheaf $\mathscr{F}_{W}$ of $\mathcal{O}_{X}$-modules on $\partial G \times W$ satisfying the following (H. 1)-(H. 3):

(H. 1) (Analytic continuation) If $U \supset V$ are open subsets of $W$ such that $U$ is connected and $V \neq \varnothing$, and if $\Omega$ is an open subset of $\partial G$, then we have

$$
\Gamma_{\Omega \times(U \backslash V)}\left(\Omega \times U, \mathscr{F}_{W}\right)=0 .
$$

(H.2) Let $f$ be a holomorphic function on $W$ with $d f \neq 0$. Put $Y=f^{-1}(0) \subset W$ and $j: \partial G \times Y \rightarrow \partial G \times W$. Then we have a short exact sequence

$$
0 \longrightarrow \mathscr{F}_{W} \stackrel{f}{\longrightarrow} \mathscr{F}_{W} \longrightarrow j_{*} \mathscr{F}_{Y} \longrightarrow 0 .
$$

(H.3) Let $W$ and $Y$ be complex manifolds with $Y$ compact. Let $q$ denote the projection from $\partial G \times W \times Y$ to $\partial G \times W$. Then

$$
R^{h} q_{*} \mathscr{F}_{W \times Y} \simeq \mathscr{F}_{W} \underset{C}{\otimes} H^{h}\left(Y, \mathcal{O}_{Y}\right) \quad(\forall h \in \mathbb{Z}) .
$$

Under the hypotheses (H.1)-(H.3) we have the following properties for $\mathscr{F}_{W}$.

(i) For any pair of holomorphically convex compact subsets $K_{1}, K_{2}$ of $\mathbb{C}^{m}$ with $K_{1}$

$\supset K_{2}$ and for any complex manifold $W$, we have

$$
H_{\partial G \times\left(K_{1} \backslash K_{2}\right) \times W}^{h}\left(\partial G \times\left(\mathbb{C}^{m} \backslash K_{2}\right) \times W, \mathscr{F}_{\mathbf{C}^{m} \times W}\right)=0(\forall h<m) .
$$


(ii) (Bochner-Kashiwara-Komatsu). For $0<\varepsilon \leqq \frac{1}{2}$, we set

$$
\begin{aligned}
G_{\varepsilon}=\left\{\left(x_{1}+i y_{1}, x_{2}+i y_{2}\right) \in \mathbf{C}^{2} ; 0 \leqq\right. & y_{1}, 0 \leqq y_{2}, y_{1}+y_{2}<1, \varepsilon\left(x_{1}^{2}+x_{2}^{2}\right) \\
& \left.+\left(y_{1}+y_{2}\right)-\varepsilon\left(y_{1}^{2}+y_{2}^{2}\right)<1-\varepsilon\right\}, \\
F_{\varepsilon}=G_{\varepsilon} \cap\left\{y_{1} y_{2}=0\right\} . &
\end{aligned}
$$

Then for any complex manifold $W$, the restriction map

$$
\Gamma\left(G_{\varepsilon} \times W, \mathscr{F}_{\mathrm{C}^{2} \times W}\right) \longrightarrow \Gamma\left(F_{\varepsilon} \times W, \mathscr{F}_{\mathrm{C}^{2} \times W}\right)
$$

is surjective.

Our $\mathscr{F}_{W}$ defined by (2.25) satisfies the conditions (H.1)-(H.3) of the above lemma, and thus it satisfies the principle of Bochner-KashiwaraKomatsu. From this the unique continuation in the variables $z^{\prime} \in \mathbf{C}^{d}$ for sections of $\mathscr{H}_{\partial G \times \mathbf{C}^{d} \times(Z \backslash D)}^{1}\left(\mathscr{F}_{\mathbf{C}^{d} \times Z}^{d}\right)$ follows. This corresponds to the unique continuation in the variables $\zeta^{\prime} \in \mathbf{C}^{d}$ for section of $C_{N \mid X}^{2}$ by $(2.24)^{\prime}$. The proof of Theorem 2.4 is complete.

Now we introduce the complex of $\mathscr{E}_{X}$-modules $\mathscr{B}_{\Omega \mid X}^{2}$ and the complex of $\pi_{L}^{-1} \mathscr{E}_{X}$-modules $\mathscr{C}_{\Omega \mid X}^{2}$ for an open subset $\Omega=\Omega^{\prime} \times L$ of $M=M^{\prime} \times L$ with $C^{\omega}$ boundary $N=N^{\prime} \times L$ (or $\Omega=M \backslash N$ with a closed $C^{\omega}$-submanifold $N=N^{\prime} \times L$ of codimension $d \geqq 2$ ).

Note that our definition of $\mathscr{B}_{\Omega \mid X}^{2}$ is different from that of [S-Z2].

We first observe that there exists a distinguished triangle

$$
\begin{array}{ll}
\mathscr{C}_{N \mid X}^{2} \longrightarrow \mathscr{C}_{M \mid X}^{2} \longrightarrow \mathscr{C}_{\Omega \mid X}^{2} \oplus \mathscr{C}_{\Omega^{-} \mid X}^{2} \stackrel{+1}{\longrightarrow}(\operatorname{cod} N=1), \\
\mathscr{C}_{N \mid X}^{2} \longrightarrow \mathscr{C}_{M \mid X}^{2} \longrightarrow \mathscr{C}_{\Omega \mid X}^{2} \stackrel{+1}{\longrightarrow} \quad(\operatorname{cod} N>1),
\end{array}
$$

where $\Omega^{-}=M \backslash \bar{\Omega}$.

Theorem 2.6. (i) $\left.\mathscr{C}_{\Omega \mid X}^{2}\right|_{T_{M}^{*}, X^{\prime} \times T_{L}^{*} Z}$ is concentrated in degree 0 . In particular $\left.\mathscr{B}_{\Omega \mid X}^{2}\right|_{T_{M}^{*}, X^{\prime} \times L}$ is concentrated in degree 0 .

(ii) $\left.\mathbf{R} \stackrel{\circ}{\pi}_{L^{*}}\left(\mathscr{C}_{\Omega \mid X}^{2}\right)\right|_{T_{M}^{*}, X^{\prime} \times L}$ is concentrated in degree $0\left(\stackrel{\circ}{\pi}_{L}: T^{*} X^{\prime} \times \stackrel{\circ}{T}_{L}^{*} Z \rightarrow T^{*} X^{\prime}\right.$ $\times L)$.

(iii) The natural morphism $\left.\left.\mathscr{C}_{\Omega \mid X}^{h}\right|_{T_{M}^{*}, X^{\prime} \times L} \rightarrow \mathscr{B}_{\Omega \mid X}^{2}\right|_{T_{M}^{*}, X^{\prime} \times L}$ is injective.

Proof. (i) follows from (2.26) and Theorem 2.4 (i). (ii), (iii): Let us apply the functor $\left.\mathbf{R} \pi_{L^{*}}^{\circ}()\right|_{T_{M}^{*}, X^{\prime} \times L}$ to (2.26). Then we have the long exact sequence 


$$
\begin{aligned}
& \left.0 \longrightarrow \mathbb{R}^{-1} \stackrel{\circ}{\pi}_{L^{*}}\left(\mathscr{C}_{\Omega \mid X}^{2}\right)_{T_{M^{*}}^{*} X^{\prime} \times L} \oplus \mathbb{R}^{-1} \stackrel{\circ}{\pi}_{L^{*}}\left(\mathscr{C}_{\Omega^{-} \mid X}^{2}\right)\right|_{T_{M^{*}}^{*}, X^{\prime} \times L} \\
& \left.\left.\longrightarrow \stackrel{\circ}{\pi}_{L^{*}}\left(\mathscr{C}_{N \mid X}^{2}\right)\right|_{T_{M^{*}}^{*} X^{\prime} \times L} \longrightarrow \stackrel{\circ}{\pi}_{L^{*}}\left(\mathscr{C}_{M \mid X}^{2}\right)\right|_{T_{M^{*}}^{*} X^{\prime} \times L} \longrightarrow \cdots .
\end{aligned}
$$

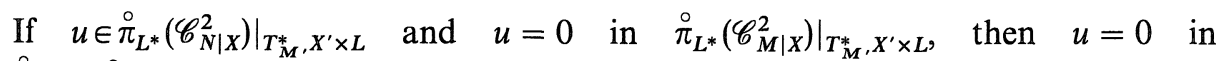
$\stackrel{\circ}{\pi}_{L^{*}}\left(\left(\mathscr{C}_{N \mid X}^{2}\right)_{T_{M}^{*}, X^{\prime} \times T_{L}^{*}, Z}\right)$ by Theorem 2.4 (i). $u$ is then zero in the whole $T_{N^{\prime}}^{*} X^{\prime}$ $\times \stackrel{\circ}{T}_{L}^{*} Z$ by Theorem 2.4 (ii). This implies

$$
\left.\mathbf{R}^{i}{\stackrel{\circ}{L^{*}}}_{\left(\mathscr{C}_{\Omega \mid X}^{2}\right.}^{2}\right)\left.\right|_{T_{M^{*}}, X^{\prime} \times L}=0 \quad(i<0) .
$$

On the other hand we have Sato's triangle for $\mathscr{C}_{\Omega \mid X}^{2}$ :

$$
\left.\left.\left.\mathscr{C}_{\Omega \mid X}^{h}\right|_{T_{M}^{*}, X^{\prime} \times L} \longrightarrow \mathscr{B}_{\Omega \mid X}^{2}\right|_{T_{M}^{*}, X^{\prime} \times L} \longrightarrow \mathbf{R}{\stackrel{\circ}{L^{*}}}\left(\mathscr{C}_{\Omega \mid X}^{2}\right)\right|_{T_{M}^{*}, X^{\prime} \times L} \stackrel{+1}{\longrightarrow}
$$

By this triangle, (ii) and (iii) follows from (i) and (ii)'.

Remark 2.7. The morphism

$$
\left.\left.\mathscr{C}_{\Omega \mid X}\right|_{T_{M}^{*}, X^{\prime} \times L} \longrightarrow \mathscr{B}_{\Omega \mid X}^{2}\right|_{T_{M}^{*}, X^{\prime} \times L}
$$

is not injective. In fact let $\operatorname{cod}_{M} N=1, \operatorname{dim} L=1$, set

$$
X^{\prime}=\mathbb{C}^{1} \times Y^{\prime} \simeq \mathbb{C}^{1} \times \mathbb{C}^{n_{1}-2} \times \mathbb{C}^{1} \ni\left(z_{1}, z^{\prime \prime}, z_{n_{1}}\right), Z \simeq \mathbb{C}^{1} \ni w
$$

and define

$$
\begin{aligned}
& U_{1}=\left\{\operatorname{Im} z_{n_{1}}>\left(\operatorname{Im} z^{\prime \prime}\right)^{2}+(\operatorname{Im} w)^{2} /\left(1-c\left(\operatorname{Im} z_{1}\right)_{+}^{2}\right)\right\}, c>0, \\
& U_{2}=\left\{\operatorname{Im} z_{n_{1}}>\left(\operatorname{Im} z^{\prime \prime}\right)^{2}+(\operatorname{Im} w)^{2}\right\},
\end{aligned}
$$

(where $\left.\left(\operatorname{Im} z_{1}\right)_{+}=\sup \left(0, \operatorname{Im} z_{1}\right)\right)$. Let $f \in \Gamma_{U_{1}}\left(\mathcal{O}_{X}\right)_{0}$; then $f$ represents a germ of $\mathscr{C}_{\Omega \mid X}$ at $\left(0 ; i d z_{n_{1}}\right)$ which is 0 in $\mathscr{B}_{\Omega \mid X}^{2}$. But $f$ is not 0 in $\mathscr{C}_{\Omega \mid X}$ as far as it does not extend holomorphically to $U_{2}$ in a neighborhood of 0 . From this and from the fact that $U_{1}, U_{2}$ are Stein, the non-injectivity of (2.27) follows.

The above remark does not affect the importance of $\mathscr{B}_{\Omega \mid X}^{2}$ at least when dealing with non-characteristic boundary value problems. In fact we have

Theorem 2.8. Let $\mathscr{M}$ be a coherent $\mathscr{E}_{X^{-}}$module at $p \in T_{N^{\prime}}^{*} X^{\prime} \times L$. Assume that $Y$ is non-characteristic for $\mathscr{M}$ at $p$. Then the morphism

$$
H^{0}\left(\mathbf{R} \mathscr{H}_{0 m_{\mathscr{E}_{X}}}\left(\mathscr{M}, \mathscr{C}_{\Omega \mid X}\right)\right)_{p} \longrightarrow H^{0}\left(\mathbf{R} \mathscr{H} \circ m_{\mathscr{E}_{X}}\left(\mathscr{M}, \mathscr{B}_{\Omega \mid X}^{2}\right)\right)_{p},
$$

is injective.

Proof. The case $d=1\left(d=\operatorname{cod}_{M} N\right)$ : Set $F=N \underset{M}{\times}\left(T_{M}^{*} X \underset{M}{\oplus} N^{*}(\Omega)^{a}\right)$ and consider the commutative diagram 


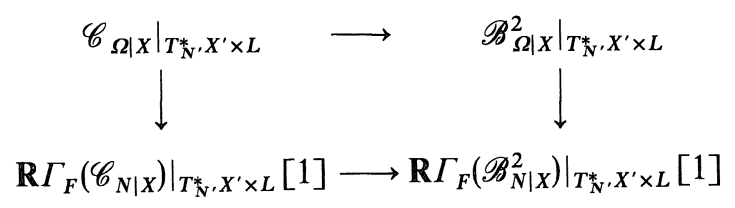

Apply the functor $\mathbf{R} \mathscr{H}_{0 m_{\mathscr{E} x}}(\mathscr{M}, \quad)$ and take the 0 -th cohomology. Then the first vertical (resp. the second horizontal) arrow becomes injective by the watermelon-cut theorem (cf. [S 3]) (resp. by the division formulas for $\mathscr{C}_{N \mid X}$ and $\mathscr{B}_{N \mid X}^{2}(=$ Lemma 2.9 below $\left.)\right)$ and by the injectivity of the morphism $\left.\mathscr{C}_{N \mid Y}\right|_{T_{M}^{*}{ }^{*}, Y^{\prime} \times L}$ $\rightarrow \mathscr{B}_{N \mid Y}^{2}$ (cf. (2.9)).

The case $d>1:$ We have

$$
H^{j}\left(\mathbf{R} \mathscr{H}_{\circ m_{\mathscr{E}_{X}}}\left(\mathscr{M}, \mathscr{C}_{N \mid X}\right)\right)=0, H^{j}\left(\mathbf{R} \mathscr{H}_{\circ m_{\mathscr{E}_{X}}}\left(\mathscr{M}, \mathscr{B}_{N \mid X}^{2}\right)\right)=0 \quad(j=0,1)
$$

by Lemma 2.9. Thus we get isomorphisms

$$
\begin{aligned}
& \mathscr{H} \circ m_{\mathscr{E}_{X}}\left(\mathscr{M}, \mathscr{C}_{M \mid X}\right)_{p} \cong H^{0} \mathbf{R} \mathscr{H}_{0 m_{\mathscr{E}_{X}}}\left(\mathscr{M}, \mathscr{C}_{\Omega \mid X}\right)_{p} . \\
& \mathscr{H} \circ{m_{\mathscr{E}_{X}}}\left(\mathscr{M}, \mathscr{B}_{M \mid X}^{2}\right)_{p} \cong H^{0} \mathbf{R} \mathscr{H}_{\circ m_{\mathscr{E}_{X}}}\left(\mathscr{M}, \mathscr{B}^{2}{ }_{\Omega \mid X}\right)_{p} .
\end{aligned}
$$

The injectivity of (2.28) then follows from the injectivity of $\left.\mathscr{C}_{M}\right|_{T_{M}^{\circ}{ }^{*}, X^{\prime} \times L} \rightarrow \mathscr{B}_{M \mid X}^{2}$ $((2.9))$. The proof is complete.

Lemma 2.9. (Division formulas for $\mathscr{C}_{N \mid X}$ and $\mathscr{B}_{N \mid X}^{2}$; cf. [K-S 1], [S-Z 1]) Let $\mathscr{M}$ be a coherent $\mathscr{E}_{X^{-}}$Module defined in a neighborhood of $p \in T_{N^{\prime}}^{*} X^{\prime}$ $\times$ L. Assume that $Y$ is non-characteristic for $\mathscr{M}$ at $p$. Then we have

$$
\begin{aligned}
& \rho_{*} \mathbf{R} \mathscr{H}_{\sigma m_{\mathscr{E}_{X}}}\left(\mathscr{M}, \mathscr{C}_{N \mid X}\right)[d] \cong \mathbf{R} \mathscr{H}_{a m_{\mathscr{E}_{Y}}}\left(\mathscr{M}_{Y}, \mathscr{C}_{N \mid Y}\right), \\
& \rho_{*} \mathbf{R} \mathscr{H}_{\circ m_{\mathscr{E}_{X}}}\left(\mathscr{M}, \mathscr{C}_{N \mid X}^{h}\right)[d] \cong \mathbf{R} \mathscr{H}_{\circ m_{\mathscr{E}} Y}\left(\mathscr{M}_{Y}, \mathscr{C}_{N \mid Y}^{h}\right) \text {, }
\end{aligned}
$$

and

$$
\rho_{*} \mathbf{R} \mathscr{H}_{\circ m_{\mathscr{E}_{X}}}\left(\mathscr{M}, \mathscr{B}_{N \mid X}^{2}\right)[d] \cong \mathbf{R} \mathscr{H} \circ m_{\mathscr{E}_{Y}}\left(\mathscr{M}_{Y}, \mathscr{B}_{N \mid Y}^{2}\right)
$$

where $\rho$ denotes the natural projection $T^{*} X \underset{X}{\times} Y \rightarrow T^{*} Y$, and $\mathscr{M}_{Y}$ denotes the tangential system of $\mathscr{M} . \quad$ In (2.30) the suffix $h$ means the holomorphicity in $w \in Z$ (see (2.1)).

Proof. (2.29) and (2.30) are proved by Kashiwara and Schapira [K$\mathrm{S}$ 1]. The formula $(2.31)$ is obtained by applying the functor $\mathbf{R} \Gamma_{T_{N}^{*}, Y^{\prime} \times L}(\quad) \otimes \omega_{L \mid Z}\left[n_{2}\right]$ to $(2.30)$.

At the end of this section we remark that our $\mathscr{B}_{\Omega \mid X}^{2}$ can be defined for some class of involutive submanifolds $V$ of $\stackrel{\circ}{T}_{M}^{*} X$.

Remark 2.10. We can define $\mathscr{B}_{\Omega \mid X}^{2}=\mathscr{B}_{\Omega \mid X}^{2, V}$ with respect to any conic involutive submanifold $V \subset \stackrel{\circ}{T}_{M}^{*} X$ such that 
(2.32) $V$ and $N \underset{M}{\times} \stackrel{\circ}{T}_{M}^{*} X$ intersect transversally and $N \underset{M}{\times} V$ is regular involutive $(N=\partial \Omega)$. In fact we can then assume, in suitable symplectic coordinates

$$
V=\stackrel{\circ}{T}_{M^{\prime}}^{*} X^{\prime} \times L, \quad \Omega \underset{M}{\times} \stackrel{\circ}{T}_{M}^{*} X=\left(\Omega^{\prime} \underset{M^{\prime}}{\times} \stackrel{\circ}{T}_{M}^{*}, X^{\prime}\right) \times T_{L}^{*} Z,
$$

and use (2.1), (2.3). On the other hand this definition is independent of the choice of the symplectic coordinates due to Theorem 2.1.

\section{$\S 3 . \Omega-V$-Microhyperbolicity}

Let $M$ be an analytic manifold of dimension $n, X$ a complexification of $M$, $\Omega$ a connected open subset of $M$. We assume that $N=\partial \Omega$ is a submanifold of $M$ of codimension $d \geqq 1$ and denote by $Y$ a complexification of $N$. Let $V$ be a conic regular involutive submanifold of $\stackrel{\circ}{T}_{M}^{*} X$ which satisfies (2.32), and let $\mathscr{B}_{M \mid X}^{2}$, $\mathscr{B}_{N \mid X}^{2}$ and $\mathscr{B}_{\Omega \mid X}^{2}$ be the complexes associated to $V$ and $\Omega \underset{M}{\times} V$ defined in $\S 2$ (cf. Remark 2.10). Let $\mathscr{E}_{X}$ be the sheaf of finite order microdifferential operators on $T^{*} X$, and let $\mathscr{M}$ be a coherent $\mathscr{E}_{X^{-}}$-module in a neighborhood of $p \in N \underset{M}{\times} V$. We will consider the problem whether

$$
\mathbb{R} \Gamma_{\pi^{-1}(N)} \mathbb{R} \mathscr{H} \operatorname{om}_{\mathscr{E}_{X}}\left(\mathscr{M}, \mathscr{B}_{\Omega \mid X}^{2}\right)_{p}=0 .
$$

The vanishing of $H^{0} \mathbb{R} \Gamma_{\pi^{-1}(N)} \mathbb{R} \mathscr{H}_{0 m_{\mathscr{E}_{X}}}\left(\mathscr{M}, \mathscr{C}_{\Omega \mid X}\right)_{p}$ (i.e., the $\Omega$-regularity of $\mathscr{M}$ at $p$ ) is already discussed by several authors (cf. e.g. [Kat], [Ô 1], [S 2], [S$Z 2]$ ). We note here that if $Y$ is non-characteristic for $\mathscr{M}$, then the vanishing of the 0 -th cohomology in (3.1) implies $\Omega$-regularity on account of Theorem 2.8. Let $x=\pi(p) . \quad X$ being the complexification of $M$, we have the embedding $T_{x}^{*} M \rightarrow T_{x}^{*} X$. Composing it with $\pi^{*}: T_{x}^{*} X \rightarrow T_{p}^{*} T^{*} X$, we have the embedding $T_{x}^{*} M \rightarrow T_{p}^{*} T^{*} X$. Let $H: T^{*} T^{*} X \rightarrow T T^{*} X$ denote the Hamiltonian isomorphism.

Theorem 3.1. Let $\Omega \subset M$ be an open connected set in a neighborhood of $x$ with analytic boundary $N$, and let $V$ be an involutive submanifold of $\stackrel{\circ}{T}_{M}^{*} X$ which verifies (2.32). Let $\mathscr{M}$ be a coherent $\mathscr{E}_{X}$-module at $p \in N \underset{M}{\times} V$, and assume that

$$
-H(\theta) \notin C_{p}\left(\operatorname{char} \mathscr{M}, \tilde{V}_{\Omega}\right), \quad \forall \theta \in\left(\stackrel{\circ}{T}_{N}^{*} M\right)_{x} \cap N_{x}^{*}(\Omega)^{a}
$$

where $\widetilde{V}_{\Omega}$ is the union of the complex bicharacteristic leaves of $V^{C}$ issued from $\bar{\Omega} \underset{M}{\times} V$ and $C($,$) is the normal cone in the sense of [K-S 1]$.

Then (3.1) holds. 
Proof. The statement is independent of the choice of a system of homogeneous symplectic coordinates of $\stackrel{\circ}{T}_{M}^{*} X$ (cf. Remark 2.10 ).

We choose symplectic coordinates such that (2.33) is fulfilled. We set $\mathscr{F}$ $=\mathbb{R} \mathscr{H}_{0 m_{\mathscr{E} X}}\left(\mathscr{M}, \mathscr{C}_{\Omega \mid X}^{h}\right)$ and observe that $\operatorname{SS}(\mathscr{F}) \subset \mathrm{C}\left(\right.$ char $\left.\mathscr{M}, \operatorname{SS}\left(\mathbb{Z}_{\Omega^{\prime} \times Z}\right)\right)$ where $\mathrm{SS}(\mathscr{F})$ denotes the microsupport of $\mathscr{F}$ in the sense of [K-S 2]. Let $d=\operatorname{cod}_{M} N$ $=1$. By (3.2) we get

$$
\operatorname{SS}(\mathscr{F})_{p} \cap\left(\stackrel{\circ}{T}_{N}^{*} M_{x} \cap N_{x}^{*}(\Omega)^{a}\right)=\varnothing .
$$

By the definition of microsupport, we have in a neighborhood of $p$

$$
\mathbb{R} \Gamma_{\pi^{-1}\left(N^{\prime} \times Z\right)} \mathbb{R} \mathscr{H} \circ m_{\mathscr{E}_{X}}\left(\mathscr{M}, \mathscr{C}_{\Omega \mid X}^{h}\right)=0 .
$$

If we then apply to (3.4) the functor $\mathbb{R} \Gamma_{T_{N}^{*}, X^{\prime} \times L}(\cdot) \otimes \omega_{L \mid Z}\left[n_{2}\right]\left(n_{2}=\operatorname{dim} L\right)$, we get (3.1).

Let $d \geqq 2$; we first note that in this case

$\widetilde{V}_{\Omega}=\widetilde{V}=$ the union of the complex bicharacteristic leaves of $V^{C}$ issued from $V$.

We need a preliminary result (valid even for $d=1$ ) whose proof is immediate.

Lemma 3.2. Let $\rho$, $\varpi$ be the canonical maps from $Y \underset{X}{\times} T^{*} X$ to $T^{*} Y$ and $T^{*} X$ respectively. Let (3.2) be fulfilled; we then have, for some neighborhood $U$ of $p$ :

$$
\begin{gathered}
\varpi^{-1}(\operatorname{char} \mathscr{M} \cap U) \cap \rho^{-1} \rho(N \underset{M}{\times} V) \subset T_{M}^{*} X, \\
\varpi^{-1}(\operatorname{char} \mathscr{M} \cap U) \cap \rho^{-1} \rho(\{p\}) \subset\{p\} .
\end{gathered}
$$

End of Proof of Theorem 3.1. Using (3.2), (3.5), (3.6), and applying Theorems 2.3.1 and 6.3.1 of [K-S 1], one easily checks that the natural morphism

$$
\mathbb{R} \mathscr{H} \operatorname{om}_{\mathscr{E}_{X}}\left(\mathscr{M}, \mathscr{C}_{N \mid X}^{h}\right) \longrightarrow \mathbb{R} \Gamma_{T_{N}^{*}, X^{\prime} \times Z} \mathbb{R} \mathscr{H} \circ m_{\mathscr{E}_{X}}\left(\mathscr{M}, \mathscr{C}_{M \mid X}^{h}\right),
$$

is a quasi-isomorphism. Hence we have

$$
\mathbb{R} \Gamma_{T_{N}^{*}, X^{\prime} \times Z} \mathbb{R} \mathscr{H} \operatorname{om}_{\mathscr{E}_{X}}\left(\mathscr{M}, \mathscr{C}_{\Omega \mid X}^{h}\right)=0 .
$$

By applying $\mathbb{R} \Gamma_{T_{N}^{*}, X^{\prime} \times L}(\cdot) \otimes \omega_{L / Z}\left[n_{2}\right]$ to (3.8), we get (3.1).

The above theorem is the 2 nd microlocal version of similar results of [Kat], [Ô 1], [S 2], [S-Z 1], and [S-Z 2].

Remark 3.3. Let $d \geqq 2$ and suppose that $Y$ is non-characteristic for $\mathscr{M}$; then we have in a neighborhood of $p$, as is shown in the proof of Theorem 2.8,

$$
\mathscr{H} o m_{\mathscr{E}_{X}}\left(\mathscr{M}, \mathscr{B}_{M \mid X}^{2}\right) \simeq H^{0} \mathbb{R} \mathscr{H} \circ m_{\mathscr{E}_{X}}\left(\mathscr{M}, \mathscr{B}_{\Omega \mid X}^{2}\right) .
$$


Suppose in addition that there exists $\theta \in\left(T_{N}^{*} M\right)_{x}(x=\pi(p))$ with $\theta \notin C_{p}$ (char $\mathscr{M}$, $\tilde{V})$. Then by (3.9) and by the microlocal Holmgren theorem due to Kashiwara (cf. $[\mathrm{K}],[\mathrm{B}]$ ), one gets the vanishing of the 0 -th cohomology of (3.1). The cohomology of degree $\geqq 1$ is not necessarily 0 in this case.

Corollary 3.4. (cf. [S-Z 1, 2]) Let $\Omega \subset M, V$ and $\mathscr{M}$ be as in Theorem 3.1. Assume that $Y$ is non-characteristic for $\mathscr{M}$ at $p$, and

$$
\left.-H(\theta) \notin \mathrm{C}_{p}\left(\operatorname{char} \mathscr{M}, \widetilde{V}_{\Omega}\right) \quad \text { (for some } \theta \in\left(\stackrel{\circ}{T}_{N}^{*} M\right)_{x} \cap N_{x}^{*}(\Omega)^{a}\right) \text {. }
$$

Then $\mathscr{M}$ is $\Omega$-regular at $p$ in the sense of [S 3], that is, the natural restriction map

$$
H^{0} \mathbf{R} \mathscr{H}_{0 m_{\mathscr{E}_{X}}}\left(\mathscr{M}, \mathscr{C}_{\Omega \mid X}\right)_{p} \longrightarrow \Gamma_{\pi^{-1}(\Omega)} \mathscr{H} \sigma m_{\mathscr{E}_{X}}\left(\mathscr{M}, \mathscr{C}_{M}\right)_{p}
$$

is injective.

Proof. The statement is independent of the choice of a system of homogeneous symplectic coordinates of $\stackrel{\circ}{T}_{M}^{*} X$ (cf. [Kat], [U 2]). Hence it is enough to prove it in the case of (2.33). Then this is a corollary of Theorem 2.8 and Theorem 3.1 (resp. Remark 3.3) for $d=1$ (resp. $d \geqq 2$ ).

Remark 3.5. In this corollary we do not need to assume that $V$ and $V \underset{M}{\times} N$ be regular, using the trick of a dummy variable due to Kashiwara. This result comprises as special cases $\Omega$-regularity of $\Omega$-hyperbolic and that of nonmicrocharacteristic systems (cf. [S 2], [S-Z 1, 2]).

Example 3.6. Let $x=\left(x_{1}, x^{\prime}, x^{\prime \prime}\right) \in M=\mathbb{R}^{n}$ with $x^{\prime}=\left(x_{2}, \cdots, x_{k}\right)$. Let $\Omega$ $=\left\{x_{1}>0\right\}$, and let $V=\left\{(x ; i \eta) \in T_{M}^{*} X \mid \eta^{\prime \prime}=0\right\}$. Let

$$
P(x, D)=D_{1}^{2}-\sum_{i, j=2}^{k} Q_{i j}\left(x_{1}^{m}, x^{\prime}\right) D_{i} D_{j}+B\left(x, D^{\prime \prime}\right)+R(x, D),
$$

where $m$ is an integer $\geq 2, Q_{i j}\left(t, x^{\prime}\right)(i, j=2, \cdots, k)$ is a real valued $C^{\omega}$-function in $\left(t, x^{\prime}\right) \in \mathbb{R}^{k}$ such that $Q_{i j}=Q_{j i}$ and the symmetric matrix $\left(Q_{i j}\left(t, x^{\prime}\right)\right)_{i, j=2}^{k}$ is positive semi-definite for any $t \geq 0$ and $x^{\prime} \in \mathbb{R}^{k-1}, B\left(x, D^{\prime \prime}\right)$ is a differential operator of the second order, and $R(x, D)$ is a lower order term. Then $\mathscr{M}=\mathscr{D}_{X} / \mathscr{D}_{X} P$ satisfies the condition (3.10).

\section{Acknowledgments}

The authors would like to express their sincere gratitude to Professor H. Komatsu and Professor K. Kataoka for giving them the opportunity of doing this work at University of Tokyo. They also thank Professor P. Schapira for his valuable suggestions. 


\section{References}

[B] Bony, J. M., Extension du Théorème de Holmgren, Sém, Goulaouic-Schwartz 19751976, Exp. 17.

[H] Hartshorne, R., Residues and duality, Lect. Notes in Math., 20, Springer, 1966.

[Kan 1] Kaneko, A., Singular spectrum of boundary values of solutions of partial differential equations with real analytic coefficients, Sci. Papers College Gen. Ed. Univ. Tokyo, 25 (1975), 59-68.

[K] Kashiwara, M., Talks in Nice, 1972.

[K-K] Kashiwara, M. and T. Kawai, On the boundary value problem for elliptic system of linear differential equations, I, Proc. Japan Acad., 48 (1972), 712-715, II, ibid., 49 (1973), 164-168.

[K-L] Kashiwara, M., and Y. Laurent, Théorème d'annulation et deuxième microlocalisation, Prepublication d'Orsay, 1983.

[K-S 1] Kashiwara, M. and P. Schapira, Microhyperbolic systems, Acta Math., 142 (1979), 1-55.

[K-S 2] - Microlocal study of sheaves, Astérisque, Soc. Math. France, 128 (1985).

[Kat] Kataoka, K., Microlocal theory of boundary value problems I, J. Fac. Sci. Univ. Tokyo Sect. IA, 27 (1980) 355-399, II, ibid., 28 (1981), 31-56.

[K-T] Kataoka, K. and N. Tose, Vanishing theorems for the sheaf of microfunctions with holomorphic parameters - Flabbiness of the sheaf of 2-microfunctions, J. Fac. Sci. Univ. Tokyo Sect. IA, 35 (1988), 313-320.

[Ko 1] Komatsu, H., Boundary values for solutions of elliptic equations, Proc. Int. Conf. Functional Analysis and Related Topics, Univ. Tokyo Press, (1970), 107-121.

[Ko 2] - A local version of Bochner's tube theorem, J. Fac. Sci. Univ. Tokyo Sect. IA, 19 (1972), 201-214.

[Ko-Kaw] Komatsu, H. and T. Kawai, Boundary values of hyperfunction solutions of linear partial differential equations, Publ. RIMS Kyoto Univ., 7 (1971), 95-104.

[Ô 1] Ôaku, T., Boundary value problems for systems of linear partial differential equations and propagation of microanaliticity, J. Fac. Sci. Univ. Tokyo Sect. IA, 33 (1986), 175-232.

[S 1] Schapira, P., Problème de Dirichlet et solutions hyperfonctions des équations elliptiques, Boll. Un. Mat. Ital., 2 (1969), 367-372.

[S 2] - Propagation at the boundary of analytic singularities, in "Singularities of Boundary Value Problems", Reidel Publ. Co., (1981), 185-212.

[S 3] - Front d'onde analytique au bord I, II, C. R. Acad Sc., 302 (1986), 383-386, and Sém. E.D.P. Ecole Polyt. Exp., 13 (1986).

[S 4] - Microfunctions for boundary value problemes, to appear.

[S-Z 1] Schapira, P. and G. Zampieri, A new class of $N$-regular systems, unpublished.

[S-Z 2] - Regularity at the boundary for systems of microdifferential equations, Pitman Research Notes in Math., 158 (1987), 186-201.

[S-K-K] Sato, M., Kawai, T. and M. Kashiwara, Hyperfunctions and pseudodifferential equations, Lect. Notes in Math., 287, Springer., (1973), 265-529.

[T] Tose, N., On a class of 2-microhyperbolic systems, J. Math. Pures et Appl., 67 (1988), 23-37.

[U 1] Uchida, M., Second microlocal boundary value problems and their applications, Master thesis, Univ. Tokyo (1987).

[U 2] - On the complex $\mathscr{C}_{A}$ attached to a certain class of Lagrangian set, Proc. Japan Acad., 64 (1988) 126-129.

[Z] Zampieri, G., An application of the 2 nd microlocalization at the boundary to the extension of solutions of differential systems, Japanese J. Math. (to appear, 1990). 
